\title{
Frequencies of Transverse and Longitudinal Oscillations in Supersonic Cavity Flows
}

\author{
Taro Handa, ${ }^{1}$ Kazuya Tanigawa, ${ }^{1}$ Yusuke Kihara, ${ }^{1}$ Hiroaki Miyachi, ${ }^{2}$ and Hatsuki Kakuno ${ }^{3}$ \\ ${ }^{1}$ Department of Energy and Environmental Engineering, Kyushu University, 6-1 Kasuga-Koen, Kasuga, Fukuoka 816-8580, Japan \\ ${ }^{2}$ Power Systems Plant Engineering Department, Mitsubishi Heavy Industries, 2-1-1 Shinhama Arai-Cho, Takasago, \\ Hyogo 676-8686, Japan \\ ${ }^{3}$ Plant and Machinery Division, Nippon Steel \& Sumikin Engineering, 46-59 Nakabaru, Tobata-Ku, Kitakyushu, \\ Fukuoka 804-8505, Japan \\ Correspondence should be addressed to Taro Handa; t.handa@kyudai.jp
}

Received 29 July 2015; Accepted 4 October 2015

Academic Editor: Keh-Chin Chang

Copyright (C) 2015 Taro Handa et al. This is an open access article distributed under the Creative Commons Attribution License, which permits unrestricted use, distribution, and reproduction in any medium, provided the original work is properly cited.

A supersonic flow over a rectangular cavity is known to oscillate at certain predominant frequencies. The present study focuses on the effect of the cavity length-to-depth $(L / D)$ ratio on the frequency for a free-stream Mach number of 1.7. The pressure oscillations are measured by changing the $L / D$ ratio from 0.5 to 3.0 , and the power spectral density is calculated from the temporal pressure signals for each $L / D$ ratio. The results demonstrate that the spectral peaks for an $L / D$ ratio of less than $\sim 1$ and greater than $\sim 2$ are accounted for by the feedback mechanisms of the transverse and longitudinal oscillations, respectively. The results also demonstrate that the spectral peaks in the transition $(1<\sim L / D<\sim 2)$ are accounted for by either of the two feedback mechanisms of transverse and longitudinal oscillations; that is, the flows under the transition regime oscillate both transversely and longitudinally.

\section{Introduction}

A supersonic flow over a rectangular cavity is known to oscillate at certain predominant frequencies. Cavity-induced pressure oscillations cause structural fatigue and sound noise. On the other hand, such oscillations are advantageous in the enhancement of supersonic mixing [1,2]; that is, the injectant is subjected to disturbances such as pressure waves or a pulsating flow generated by a cavity-induced flow oscillation. A linear stability analysis provides the principle in which the growth rate of a supersonic shear-layer depends on the frequency of the disturbance imposed on the shear layer [3]. Hence, it is important for an enhancement of the supersonic mixing to estimate the frequency of a cavity-induced flow oscillation.

According to Rockwell and Naudascher [4], cavities can be classified into two types with respect to the oscillation direction (direction of pressure-wave propagation) inside the cavity. In general, a cavity is called a "shallow cavity" when the flow oscillates predominantly in a longitudinal direction and a "deep cavity" when the flow oscillates predominantly in a transverse direction. According to Rockwell and Naudascher [4], the transition between transverse and longitudinal oscillations occurs at $L / D \sim 1$.

The feedback mechanism of longitudinal oscillation can be expressed as Rossiter's model [5] or modified Rossiter's model [6]. Using such models, the predominant oscillation frequencies can be estimated rather accurately for shallow cavities, but the estimation fails for deeper cavities because the effect of the cavity depth is not included in these models. Based on a flow visualization, Handa et al. [7] recently developed a model accounting for the feedback mechanism of a transverse oscillation [8]. Their model estimates the dominant frequencies well for deep cavities whose lengthto-depth ratios are lower than 1.0. Before the development of their model, it had been believed for a long time that the empirical formula of East [9] could be used to estimate the predominant oscillation frequencies for supersonic deepcavity flows. However, to the best of our knowledge, East's formula cannot reproduce any experimental results for supersonic deep-cavity flows. 


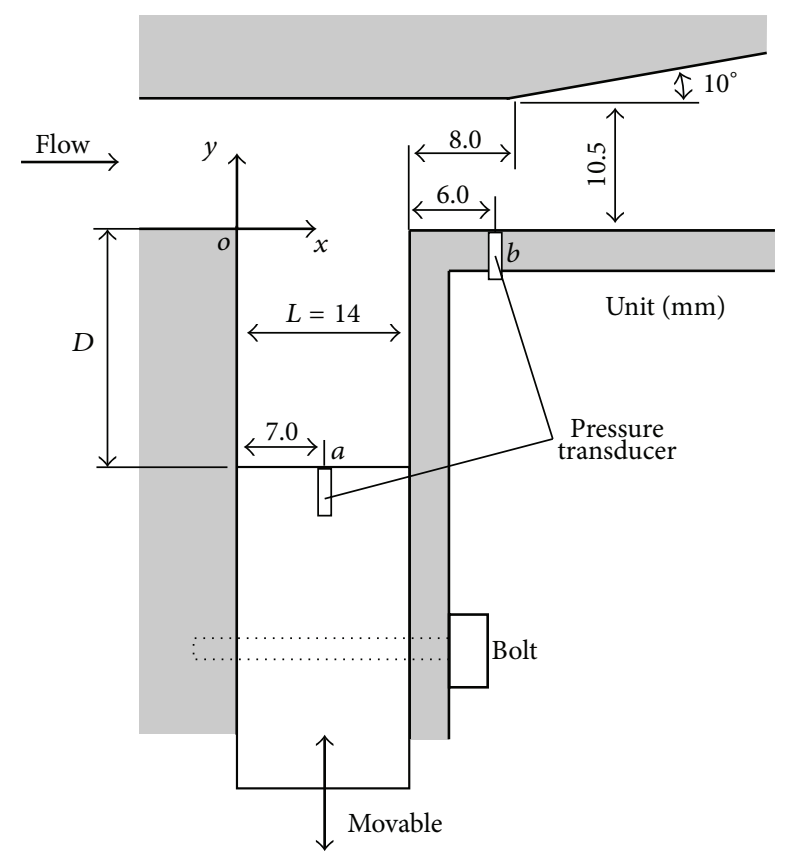

FIgURE 1: Test duct.

When the $L / D$ ratio is increased from a deep to shallow cavity, a transition from a transverse oscillation into a longitudinal oscillation should occur. However, it remains unclear how a flow oscillates under the transition regime because the oscillation frequencies have yet to be discussed in detail under the transition regime; this is due to the fact that, until the formula proposed by Handa et al., there were no formulae developed for estimating the oscillation frequencies for transverse oscillations. In the present study, the pressure oscillations are measured by changing the $L / D$ ratio between 0.50 and 3.0. Peaks appearing in the pressure oscillation spectra are assigned to the oscillation modes accounted for by either of the two feedback mechanisms of transverse and longitudinal oscillations; further, how the oscillation frequencies change with the $L / D$ ratio is discussed herein.

\section{Experiments}

Figure 1 shows the detailed structure of the test duct. Nitrogen gas flows into the test duct through a nozzle at a Mach number of 1.68. Detailed descriptions of the flow issuing from the nozzle are provided elsewhere $[7,8]$; on the basis of the boundary layer thickness, the flow issuing from the nozzle results in a boundary layer thickness of $0.73 \mathrm{~mm}$ and a Reynolds number of $1.03 \times 10^{4}$. The test duct has a rectangular cross section with a height and width of 10.5 and $28.0 \mathrm{~mm}$, respectively. With a width of $28.0 \mathrm{~mm}$, the cavity spans the full width of the duct. The cavity length $L$ is $14.0 \mathrm{~mm}$, whereas the depth $D$ is adjustable. In the present experiments, $D$ is varied from 4.7 to $28.0 \mathrm{~mm}$, which implies that the $L / D$ ratio varies from 0.50 to 3.0 .

The temporal variations in the pressure are measured using semiconductor-type pressure transducers (Kulite,
XCQ-062). One transducer is mounted on the bottom wall of the cavity, $7.0 \mathrm{~mm}$ downstream from the front wall of the cavity (location $a$ in Figure 1). The other transducer is mounted on the lower duct wall, $6.0 \mathrm{~mm}$ downstream of the trailing edge (location $b$ in Figure 1). Both transducers are placed in the central spanwise plane of the duct. The temporal pressure signals are recorded using a digital oscilloscope (IWATSU DS-9121) at a sampling rate of $500 \mathrm{k}$ samples per second for a total of 65536 samples. The power spectral density is calculated from the temporal variation of the pressure signal. Ten experimental runs were conducted under the same flow conditions. A dominant peak appears in a power spectrum for each $L / D$ ratio. The dominant peak frequency at location $a$ is identical to that at location $b$ for almost all $L / D$ ratios, although several minor peaks appearing in the spectra at location $b$ do not appear in the spectra at location $a$. Hence, in this study, we show the pressure oscillation spectra at only location $b$. Several studies $[2,10]$ demonstrated that the injection just downstream of the cavity was effective in the enhancement of supersonic mixing. Information about the pressure oscillation at location $b$ might give significance to the mixing enhancement. We confirmed that the frequencies of the dominant spectral peaks in ten runs agreed within $\pm 200 \mathrm{~Hz}$ (within \pm 0.006 in Strouhal number). During the experiments, the pressure in the stagnation chamber was maintained at $101 \mathrm{kPa}$, and the chamber was kept at atmospheric temperature (which was monitored during each experimental run).

\section{Feedback Mechanisms}

In each spectrum of the pressure oscillation measured in this study, several peaks were observed. Almost all of the peaks originated from feedback mechanisms for the transverse [7] and longitudinal oscillations $[5,6]$, although a few peaks originated from an acoustic resonance. In the following paragraphs, the feedback mechanisms for both oscillations are briefly reviewed.

Rossiter [5] visualized a cavity flow using a shadowgraph technique and found that a shear layer is generated at the leading edge of the cavity and that a discrete vortical structure is convected downstream within the shear layer. Based on this visualization, Rossiter developed an oscillation model for a flow. According to this model, a pressure wave is produced when a vortical structure impinges on the trailing edge of the cavity, and this wave then propagates upstream inside the cavity. When the pressure wave reaches the leading edge of the cavity, another vortical structure is created, which is then convected downstream to the trailing edge of the cavity. A feedback loop is thus completed, and the pressure oscillation is sustained. For such a feedback mechanism, Rossiter derived the following formula:

$$
\mathrm{St}=\frac{f L}{U_{\infty}}=\frac{n-r}{M_{\infty}+1 / k},
$$

where St is the Strouhal number, $f$ is the oscillation frequency, $U_{\infty}$ is the free-stream velocity, $M_{\infty}$ is the free-stream Mach number, $n$ is the oscillation mode number, $r$ is the phase 
delay constant, and $k$ is the ratio of the convection velocity of a vortical structure $U_{c}$ to $U_{\infty}$. In the feedback mechanism expressed by Rossiter's model, the pressure wave propagates longitudinally. Hence, the oscillation expressed by Rossiter's model is called "longitudinal oscillation."

Equation (1) is formulated based on the assumption that the speed of sound inside a cavity is equal to that within a free stream. However, this assumption leads to errors at high Mach-number flows, and to overcome such difficulty, Heller et al. [6] assume that the speed of sound inside a cavity $a_{\text {cavity }}$ is equal to that in a stagnating free stream:

$$
a_{\text {cavity }}=a_{\infty}\left(1+\frac{\gamma-1}{2} M_{\infty}^{2}\right)^{1 / 2},
$$

where $a_{\infty}$ is the speed of sound in a free stream and $\gamma$ is the specific heat ratio. Equation (1) can then be rewritten as

$$
\mathrm{St}=\frac{f L}{U_{\infty}}=\frac{n-r}{M_{\infty}\left\{1+[(\gamma-1) / 2] M_{\infty}^{2}\right\}^{-1 / 2}+1 / k} .
$$

Note that this equation does not include the wave reflection at the bottom wall of the cavity. The constants $k$ and $r$ were empirically determined by Rossiter to be 0.57 and 0.25 , respectively [5].

A longitudinal oscillation in supersonic shallow-cavity flows is expressed by the modified Rossiter formula in (3). Using this formula, the predominant frequencies can be estimated rather accurately for shallow cavities, whereas the estimation fails for deeper cavities because the effect of the cavity depth is not included. Handa et al. [7] recently proposed an oscillation model for estimating the predominant frequencies of the pressure oscillation in a supersonic deepcavity flow:

$$
\mathrm{St}=\frac{n-r-\Delta \phi / 2 \pi}{\left(M_{\infty} / \alpha_{I}\right)\left\{1+[(\gamma-1) / 2] M_{\infty}^{2}\right\}^{-1 / 2}+1 / k},
$$

where $\Delta \phi$ is expressed in detail in [7]. The pressure-wave propagation inside a deep cavity was visualized in a time sequence of the schlieren images captured by Handa et al. [8]. According to their visualization results, two pressure waves play an important role in the feedback mechanism: one wave is generated at the trailing edge of the cavity owing to the motion of the shear layer, and the other wave is generated as a result of the third reflection of the former wave at the bottom of the cavity; $\Delta \phi$ in (4) can be calculated by modeling these two waves. From the schlieren images, it was found that the latter wave propagates transversely, and thus the flow oscillation accounted for by (4) is considered to be a "transverse oscillation."

The constants $k, r$, and $\alpha_{I}$ in (4) are given as 0.76, 0.26 , and 0.75 , respectively. Handa et al. [7] discussed the universality of these constants by comparing the calculation results with the experimental results for deep cavities $(L / D \leq$ 1) measured by the other researchers, and they concluded that the constants are useful for Mach numbers ranging between 1.5 and 2.5, for the width to depth ratio of the cavity $W / D$ ranging between 0.36 and 2.1, and for Reynolds numbers

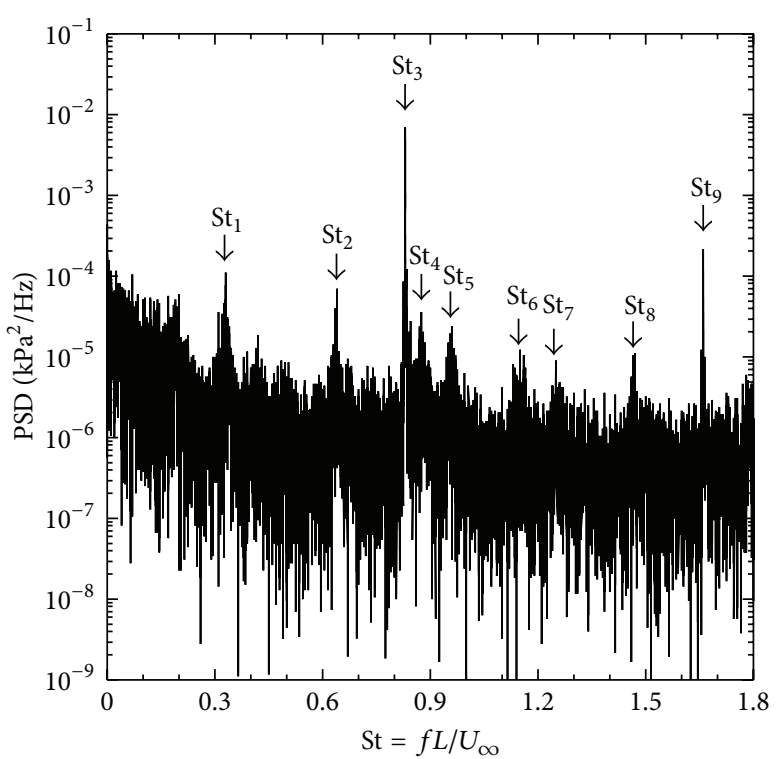

Figure 2: Power spectral density of pressure oscillation for $L / D=$ $0.70[7]$.

(based on the boundary layer thickness) $\mathrm{Re}_{b}$ ranging between $9.86 \times 10^{3}$ and $2.16 \times 10^{5}$; that is, the results calculated from (4) agreed with the experimental results to within $4 \%$.

Acoustic waves propagating in the spanwise direction may potentially affect the feedback mechanism. However, the agreement between calculated and experimental results for values of $W / D$ ranging widely between 0.36 and 2.1 implies that the feedback mechanism may not be influenced by acoustic waves propagating in the spanwise direction. Incoming boundary-layer thickness may be also a dominant factor potentially. The agreement between calculated and experimental results for values of $\mathrm{Re}_{b}$ ranging widely between $9.86 \times 10^{3}$ and $2.16 \times 10^{5}$ implies that incoming boundarylayer thickness may not be a dominant factor for the feedback mechanism.

\section{Results and Discussion}

Figure 2 shows the power spectrum of the pressure oscillation measured for $L / D=0.70$. This spectrum is the same as that shown in [7]. The horizontal axis indicates the value of St calculated based on $L$ and $U_{\infty}$. According to Rockwell and Naudascher [4], the cavity for $L / D=0.70$ is classified as a deep cavity. In Figure 2, a single strong peak and several weak peaks can be seen. The spectral peaks appearing near the Strouhal numbers calculated from (3) (longitudinal oscillation) and (4) (transverse oscillation) are listed in Tables $1(\mathrm{a})$ and $1(\mathrm{~b})$, respectively. The corresponding peaks marked in the spectra of Figure 2 are noted by the St labels in the parentheses, and the dominant peak is denoted in boldface. We found only one small spectral peak, $\mathrm{St}_{4}$ within \pm 0.02 of the Strouhal number calculated from (3) for $n=3$, and four spectral peaks, $\mathrm{St}_{2}, \mathrm{St}_{3}, \mathrm{St}_{6}$, and $\mathrm{St}_{7}$ (the dominant peak being $\mathrm{St}_{3}$ ) within \pm 0.02 of the Strouhal numbers calculated from (4) for $n=2-4$. These results imply that the flow for $L / D=0.70$ 
TABLE 1: Strouhal numbers of pressure oscillations resulting from feedback mechanism for $L / D=0.70$.

(a) Equation (3) (longitudinal oscillation)

\begin{tabular}{lccccc}
\hline & $n=1$ & $n=2$ & $n=3$ & $n=4$ & $n=5$ \\
\hline St (Cal.) & 0.24 & 0.57 & 0.89 & 1.21 & 1.53 \\
St (Exp.) & - & - & $0.88\left(\mathrm{St}_{4}\right)$ & - & - \\
\hline
\end{tabular}

(b) Equation (4) (transverse oscillation)

\begin{tabular}{lcccccccc}
\hline & $n=1$ & $n=2$ & $n=2$ & $n=3$ & $n=3$ & $n=4$ & $n=4$ & $n=5$ \\
\hline St (Cal.) & 0.24 & 0.54 & 0.64 & 0.83 & 0.93 & 1.13 & 1.23 \\
St (Exp.) & - & - & $0.64\left(\mathrm{St}_{2}\right)$ & $\mathbf{0 . 8 3}\left(\mathbf{S t}_{3}\right)$ & - & $1.15\left(\mathrm{St}_{6}\right)$ & $1.25\left(\mathrm{St}_{7}\right)$ & - \\
\hline
\end{tabular}

oscillates primarily by the feedback mechanism accounted for by the model by Handa et al. (4); that is, the flow primarily oscillates transversely.

A few weak peaks other than those listed in the tables were detected in the spectrum, no detailed descriptions of which are given in [7]. One of these peaks, $\mathrm{St}_{1}(=0.33)$, may have resulted from the acoustic resonance. If standing waves appear in the cavity along the longitudinal direction, the Strouhal number is given by

$$
\mathrm{St}=\frac{f L}{U_{\infty}}=\frac{m a_{u} a_{d}}{U_{\infty}\left(a_{u}+a_{d}\right)}, \quad m=1,2, \ldots,
$$

where $a_{u}$ and $a_{d}$ are the speeds of the upstream and downstream propagating waves, respectively. The upstream and downstream propagating waves were clearly visualized in a time-sequence of the schlieren flow images for $L / D=0.70$ [8]. By carefully tracking these waves in the images, we obtain $a_{u}=266 \mathrm{~m} / \mathrm{s}$ and $a_{d}=379 \mathrm{~m} / \mathrm{s}$. The Strouhal number can now be calculated from (5) as St $=0.33$ for $m=1$, which agrees with the Strouhal number of peak $\mathrm{St}_{1}(=0.33)$. The other peaks may result from an amplitude modulation [11]. Actually, $\mathrm{St}_{8}$ and $\mathrm{St}_{9}$ in Figure 2 are expressed as $\mathrm{St}_{2}+\mathrm{St}_{3}$ and $2 \mathrm{St}_{3}\left(=\mathrm{St}_{3}+\mathrm{St}_{3}\right)$, respectively. Spectral peak $\mathrm{St}_{5}(=0.96)$ cannot be assigned to any oscillation modes, although this peak appears somewhat near the Strouhal number calculated from (4) as the higher of the two modes at $n=3(\mathrm{St}=0.93)$.

Figure 3 shows the power spectrum of the pressure oscillation measured for $L / D=2.8$. According to Rockwell and Naudascher [4], the cavity for $L / D=2.8$ is classified as a shallow cavity. Similar to Figure 2, a single strong peak and two weak peaks (although detectable) can be seen. The spectral peaks appearing within \pm 0.02 of the Strouhal numbers calculated from (3) (longitudinal oscillation) are listed in Table 2. The peaks $\mathrm{St}_{1}$ and $\mathrm{St}_{2}$ correspond to the modes of $n=1$ and 2, respectively. No peaks appear near the Strouhal numbers calculated from (4) (transverse oscillation). These results are the same as those observed in numerous experimental and computational studies for supersonic shallow-cavity flows $[6,12-14]$; that is, the flow for $L / D=2.8$ oscillates longitudinally. The peak $\mathrm{St}_{3}$ in Figure 3 should appear as a result of the amplitude modulation because $\mathrm{St}_{3}$ is expressed by $2 \mathrm{St}_{2}$.

Figure 4 shows the power spectrum of the pressure oscillations measured for $L / D=1.8$. The spectral peaks appearing within \pm 0.02 of the Strouhal numbers calculated from (3)
TABLE 2: Strouhal numbers of pressure oscillations resulting from a feedback mechanism calculated from (3) (longitudinal oscillation) for $L / D=2.8$.

\begin{tabular}{lcccc}
\hline & $n=1$ & $n=2$ & $n=3$ & $n=4$ \\
\hline St (Cal.) & 0.24 & 0.57 & 0.89 & 1.21 \\
St (Exp.) & $0.26\left(\mathrm{St}_{1}\right)$ & $\mathbf{0 . 5 8}\left(\mathbf{S t}_{\mathbf{2}}\right)$ & - & - \\
\hline
\end{tabular}

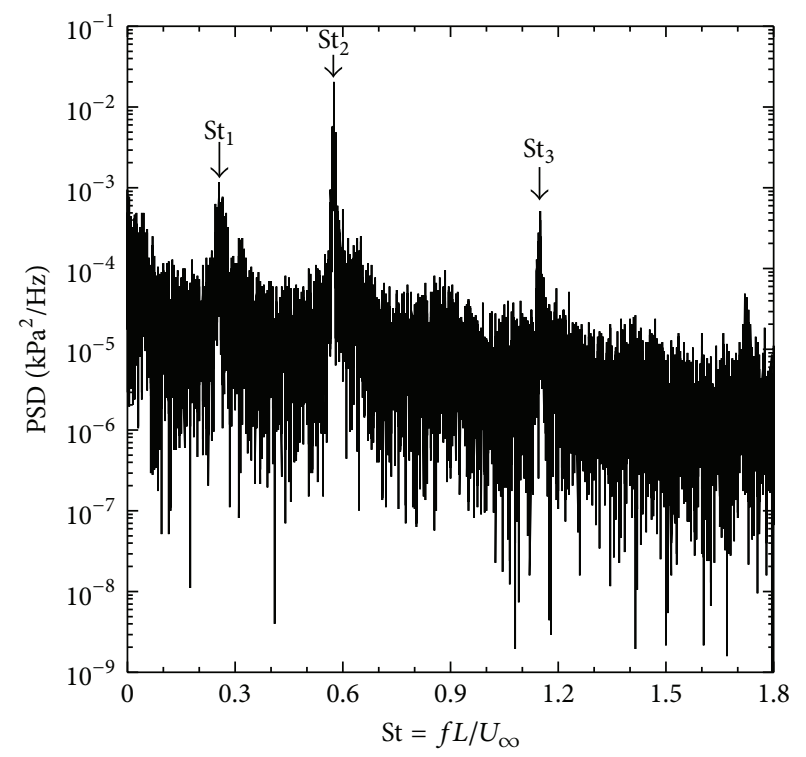

FIGURE 3: Power spectral density of pressure oscillation for $L / D=$ 2.8 .

(longitudinal oscillation) and (4) (transverse oscillation) are listed in Table 3. The spectral peaks in Figure 4 are assigned not only to a longitudinal oscillation $\left(\mathrm{St}_{2}, \mathrm{St}_{4}, \mathrm{St}_{5}\right.$, and $\left.\mathrm{St}_{7}\right)$ but also to a transverse oscillation $\left(\mathrm{St}_{1}\right.$ and $\left.\mathrm{St}_{3}\right)$. This finding implies that the flow for $L / D=1.8$ oscillates not only longitudinally but also transversely. The weak peak, $\mathrm{St}_{6}$ $(=1.27)$, might appear as a result of the amplitude modulation because $\mathrm{St}_{6}$ is expressed by $\mathrm{St}_{7}-\mathrm{St}_{1}$.

We tried to visualize the pressure waves traveling inside the cavity of $L / D=1.8$ using the schlieren technique with a spark light source whose duration time was $\sim 30 \mathrm{~ns}$. However, no pressure waves appeared inside the cavity in the instantaneous schlieren images although the waves were clearly visualized for a deep cavity of $L / D=0.70$ [8]. 
TABLE 3: Strouhal numbers of pressure oscillations resulting from feedback mechanisms for $L / D=1.8$.

(a) Equation (3) (longitudinal oscillation)

\begin{tabular}{lccccc}
\hline & $n=1$ & $n=2$ & $n=3$ & $n=4$ & $n=5$ \\
\hline St (Cal.) & 0.24 & 0.57 & 0.89 & 1.21 & 1.53 \\
St (Exp.) & - & $\mathbf{0 . 5 6}\left(\mathbf{S t}_{\mathbf{2}}\right)$ & $0.90\left(\mathrm{St}_{4}\right)$ & $\mathbf{1 . 2 2}\left(\mathbf{S t}_{\mathbf{5}}\right)$ & $1.55\left(\mathrm{St}_{7}\right)$ \\
\hline
\end{tabular}

(b) Equation (4) (transverse oscillation)

\begin{tabular}{lccccccc}
\hline & $n=1$ & $n=1$ & $n=2$ & $n=2$ & $n=3$ & $n=3$ & $n=4$ \\
\hline St (Cal.) & 0.17 & 0.28 & 0.51 & 0.62 & 0.84 & 0.96 & 1.18 \\
St (Exp.) & - & $0.28\left(\mathrm{St}_{1}\right)$ & - & $0.62\left(\mathrm{St}_{3}\right)$ & - & - & - \\
\hline
\end{tabular}

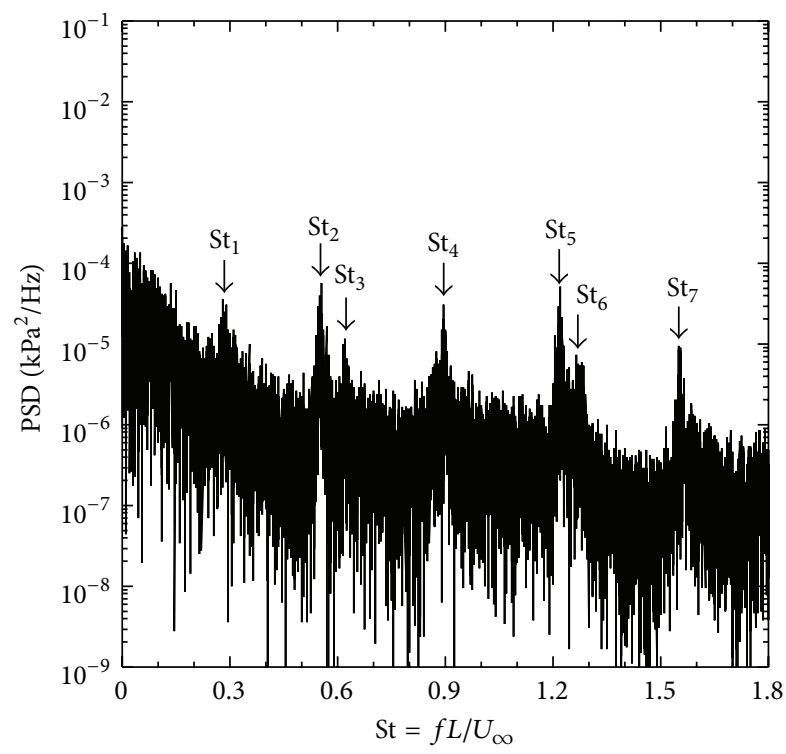

Figure 4: Power spectral density of pressure oscillation for $L / D=$ 1.8 .

Chandra and Chakravarthy [15] also captured instantaneous schlieren images of Mach 1.5 flows over a rectangular cavity of $L / D=1.5$ (which is close to $L / D=1.8$ ). Similar to our visualization results, no pressure waves appeared inside the cavity in their schlieren images although the waves were visualized for a deep cavity of $L / D=0.57$. Li et al. [16] recently conducted the numerical simulations for Mach 2 and 3 flows over a rectangular cavity of $L / D=2.0$ (which is close to $L / D=1.8$ ). By calculating the divergence of velocity, they successfully visualized the pressure waves propagating inside the cavity. Their visualization results demonstrated that the waves propagated not only longitudinally but also transversely even when the cavity was not deep $(L / D>\sim$ 1), which strongly supports our findings stated in the last paragraph; that is, the flow for $L / D=1.8$ oscillates not only transversely but also longitudinally.

The Strouhal numbers of the detectable spectral peaks are plotted against the $L / D$ ratio in Figure 5. The Strouhal numbers calculated from (3), (4), and (5) are also shown by dashed, solid, and dotted lines in the figure, respectively. The data corresponding to the spectral peaks resulting from an amplitude modulation are not plotted in the figure. As

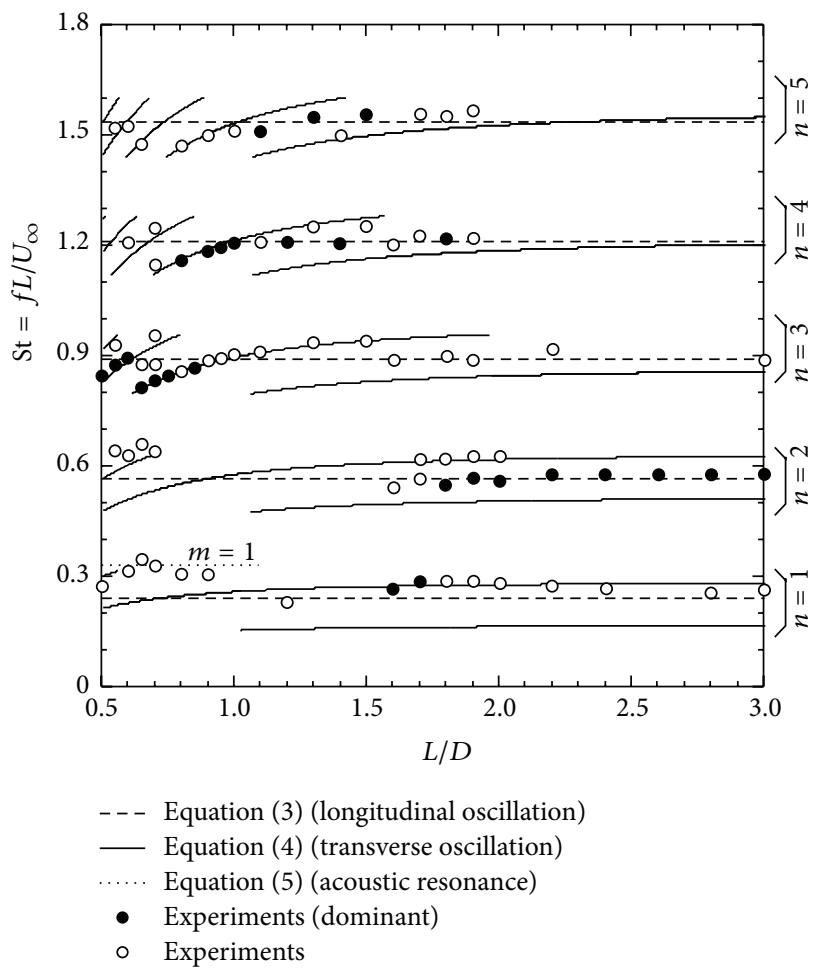

FIGURE 5: Plots of Strouhal number St versus $L / D$.

mentioned above, the oscillations accounted for by (3) and (4) correspond to longitudinal and transverse oscillations, respectively. As shown in Figure 5, the model by Handa et al. (4) estimates the Strouhal numbers of the spectral peaks (including the dominant peaks) well for $L / D<\sim$ 1 although a few peaks obey (3) (longitudinal oscillation) or (5) (acoustic resonance), whereas the modified Rossiter's model (3) performs well for $L / D>\sim 2$. Furthermore, the Strouhal numbers of the spectral peaks for $1<\sim L / D<\sim 2$ are estimated by either (3) or (4). These findings imply that the transition from a transverse oscillation to a longitudinal oscillation occurs between $L / D \sim 1$ and $\sim 2$ and that the flows under the transition regime oscillate both transversely and longitudinally.

The root-mean-square value of pressure fluctuation $\Delta p_{\text {rms }}$ is calculated and plotted against the $L / D$ ratio as shown in Figure 6. In the calculation of $\Delta p_{\text {rms }}$, the spectral components 


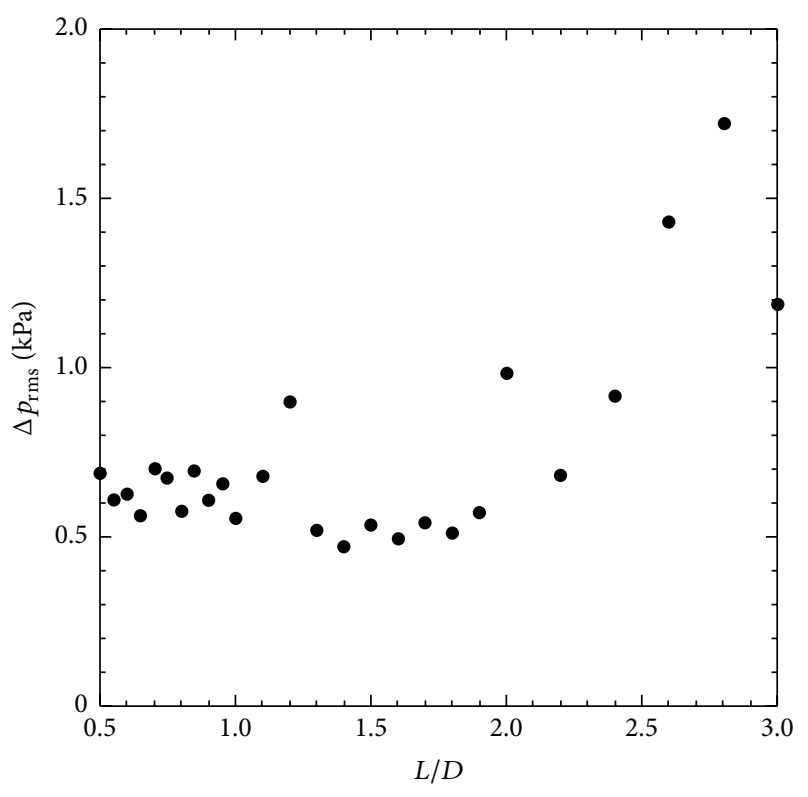

Figure 6: Plots of $\Delta p_{\text {rms }}$ (location $b$ ) versus $L / D$.

appearing around the natural frequency of the pressure transducer are eliminated using the numerical filter (a peak appears in the pressure oscillation spectra at $\sim 170 \mathrm{kHz}$ or $~$ $190 \mathrm{kHz}$ because a pressure transducer has been replaced with a broken transducer). Larchevêque et al. [17] mentioned such natural frequency components. They, however, demonstrated that the pressure-oscillation spectrum obtained by the largeeddy simulation (LES) agreed very well with that measured by Forestier et al. [18] except the spectral components around the natural frequency of the pressure transducer. That is why reasonably correct values of $\Delta p_{\text {rms }}$ can be estimated by eliminating the components around the natural frequency. It is found from Figure 6 that $\Delta p_{\mathrm{rms}}$ is almost constant for $L / D<\sim$ 2 and $\Delta p_{\text {rms }}$ increases with increase in $L / D$ for $L / D>\sim 2$. These findings imply that the pressure oscillation begins to intensify when the transition from transverse to longitudinal oscillation has terminated. Chandra and Chakravarthy [15] showed plots similar to Figure 6. In their plots, the pressure oscillation intensifies not only for shallow cavities $(L / D \sim$ $2.25)$ but also for deep cavities $(L / D \sim 0.75)$. Although we have no clear reason for the difference between their and our results, both results imply that the pressure oscillation intensifies when the transverse oscillation has disappeared. It is expected that the transverse oscillation may possibly act on the flow so that the longitudinal oscillation is suppressed although the detailed mechanism behind the suppression remains unclear at present.

The Strouhal numbers based on the cavity depth have also been calculated and plotted against the $L / D$ ratio (the results are not shown). However, we cannot find out any specific characteristics; that is, the Strouhal number varies with $L / D$. If the cavity depth was the characteristic length in the cavityinduced flow oscillations, the Strouhal number attained a constant value in a certain range of $L / D$.

\section{Conclusions}

The pressure oscillations in gaseous flows over a rectangular cavity at a Mach number of 1.7 were measured when changing the cavity length-to-depth $(L / D)$ ratio from 0.5 to 3.0. The power spectral density was calculated from the temporal pressure signal for each $L / D$ ratio.

Almost all spectral peaks including dominant peaks for $L / D<\sim 1$ were accounted for by the feedback mechanism of transverse oscillation that were recently clarified, although a few weak peaks were accounted for by an acoustic resonance or the feedback mechanism of longitudinal oscillation. Spectral peaks for $L / D>\sim 2$ were accounted for by a traditional way of thinking, that is, the feedback mechanism of longitudinal oscillation (Rossiter's model). It was determined in this study that the transition between transverse and longitudinal oscillations occurred between $L / D \sim 1$ and $\sim 2$ and that the spectral peaks in the transition were accounted for by either of the two feedback mechanisms of transverse and longitudinal oscillations. It was therefore concluded that the flows under the transition regime oscillate both transversely and longitudinally.

\section{Conflict of Interests}

The authors declare that there is no conflict of interests regarding the publication of this paper.

\section{References}

[1] K. H. Yu and K. C. Schadow, "Cavity-actuated supersonic mixing and combustion control," Combustion and Flame, vol. 99, no. 2, pp. 295-301, 1994.

[2] T. Handa, A. Nakano, K. Tanigawa, and J. Fujita, "Supersonic mixing enhanced by cavity-induced three-dimensional oscillatory flow," Experiments in Fluids, vol. 55, no. 4, article 1711, 2014.

[3] T. L. Jackson and C. E. Grosch, "Inviscid spatial stability of compressible mixing layer," Journal of Fluid Mechanics, vol. 208, pp. 609-637, 1989.

[4] D. Rockwell and E. Naudascher, "Review—self-sustaining oscillations of flow past cavities," Journal of Fluids Engineering, vol. 100, no. 2, pp. 152-165, 1978.

[5] J. E. Rossiter, Wind-Tunnel Experiments on the Flow over Rectangular Cavities at Subsonic and Transonic Speeds, Reports and Memoranda no. 3488, Aeronautical Research Council, 1964.

[6] H. H. Heller, D. G. Holmes, and E. E. Covert, "Flow-induced pressure oscillations in shallow cavities," Journal of Sound and Vibration, vol. 18, no. 4, pp. 545-553, 1971.

[7] T. Handa, H. Miyachi, H. Kakuno, T. Ozaki, and S. Maruyama, "Modeling of a feedback mechanism in supersonic deep-cavity flows," AIAA Journal, vol. 53, no. 2, pp. 420-425, 2015.

[8] T. Handa, H. Miyachi, H. Kakuno, and T. Ozaki, "Generation and propagation of pressure waves in supersonic deep-cavity flows," Experiments in Fluids, vol. 53, no. 6, pp. 1855-1866, 2012.

[9] L. F. East, "Aerodynamically induced resonance in rectangular cavities," Journal of Sound and Vibration, vol. 3, no. 3, pp. 277$287,1966$. 
[10] V. A. Nenmeni and K. Yu, "Cavity-induced mixing enhancement in confined supersonic flows," AIAA-Paper 2002-1010, 2002.

[11] N. Delprat, "Rossiter's formula: a simple spectral model for a complex amplitude modulation process?" Physics of Fluids, vol. 18, no. 7, Article ID 071703, 2006.

[12] X. Zhang and J. A. Edwards, "An investigation of supersonic oscillatiory cavity flows driven by thick shear layers," Aeronautical Journal, vol. 94, no. 940, pp. 355-364, 1990.

[13] Ö. H. Ünalmis, N. T. Clemens, and D. S. Dolling, "Cavity oscillation mechanisms in high-speed flows," AIAA Journal, vol. 42, no. 10, pp. 2035-2041, 2004.

[14] H. Wang, M. Sun, N. Qin, H. Wu, and Z. Wang, "Characteristics of oscillations in supersonic open cavity flows," Flow, Turbulence and Combustion, vol. 90, no. 1, pp. 121-142, 2013.

[15] B. U. Chandra and S. R. Chakravarthy, "Experimental investigation of cavity-induced acoustic oscillations in confined supersonic flow," Journal of Fluids Engineering, vol. 127, no. 4, pp. 761-769, 2005.

[16] W. Li, T. Nonomura, A. Oyama, and K. Fujii, "Feedback mechanism in supersonic laminar cavity flows," AIAA Journal, vol. 51, no. 1, pp. 253-257, 2013.

[17] L. Larchevêque, P. Sagaut, I. Mary, O. Labbé, and P. Comte, "Large-eddy simulation of a compressible flow past a deep cavity," Physics of Fluids, vol. 15, no. 1, pp. 193-210, 2003.

[18] N. Forestier, L. Jacquin, and P. Geffroy, "The mixing layer over a deep cavity at high-subsonic speed," Journal of Fluid Mechanics, vol. 475, no. 1, pp. 101-145, 2003. 

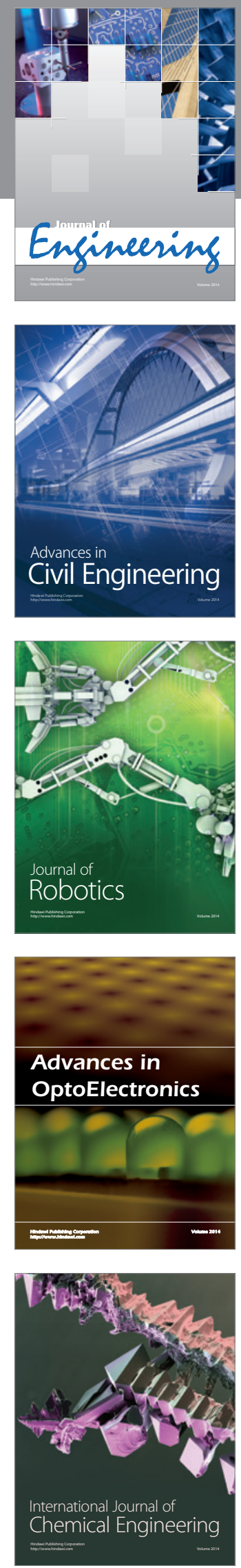

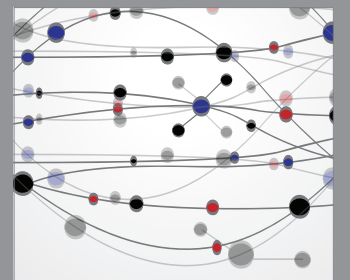

The Scientific World Journal
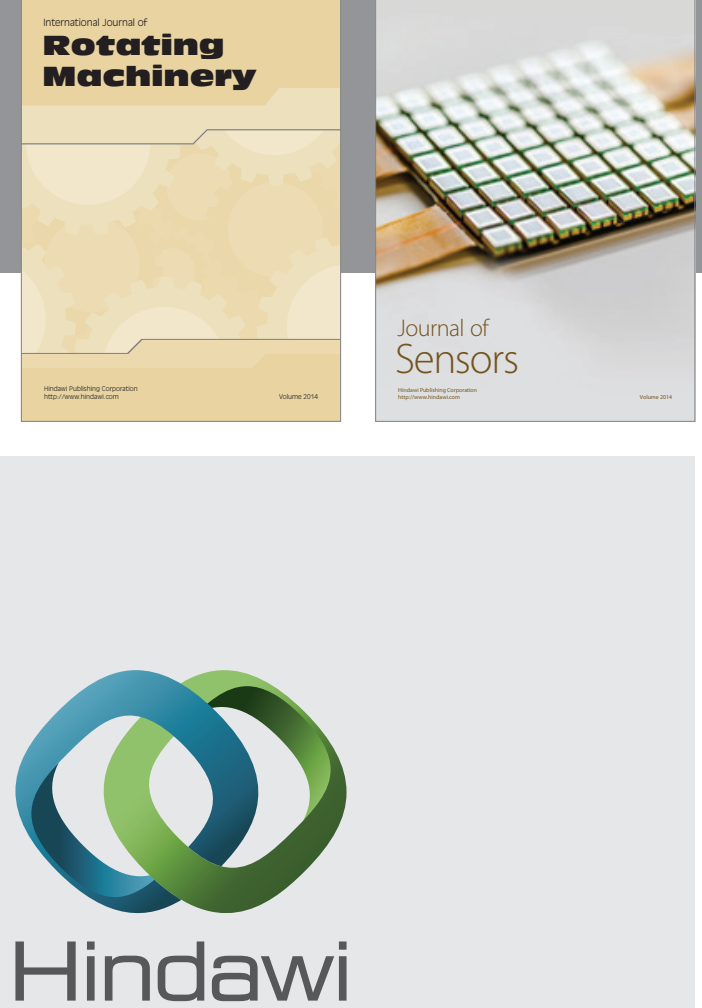

Submit your manuscripts at http://www.hindawi.com
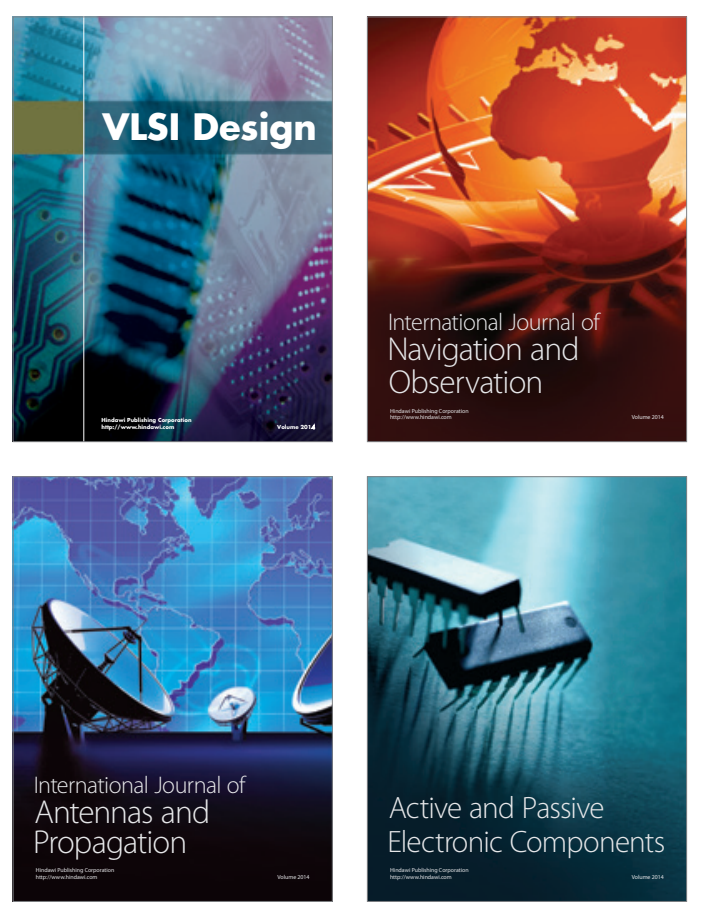
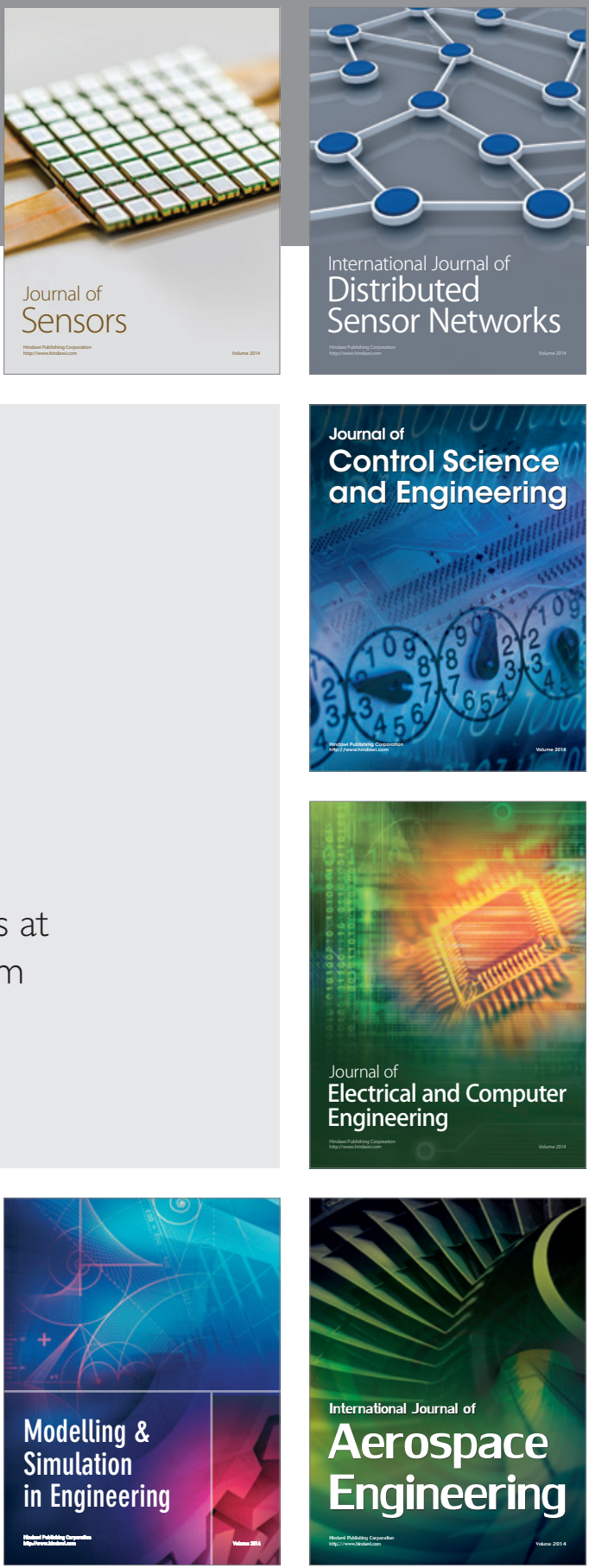

Journal of

Control Science

and Engineering
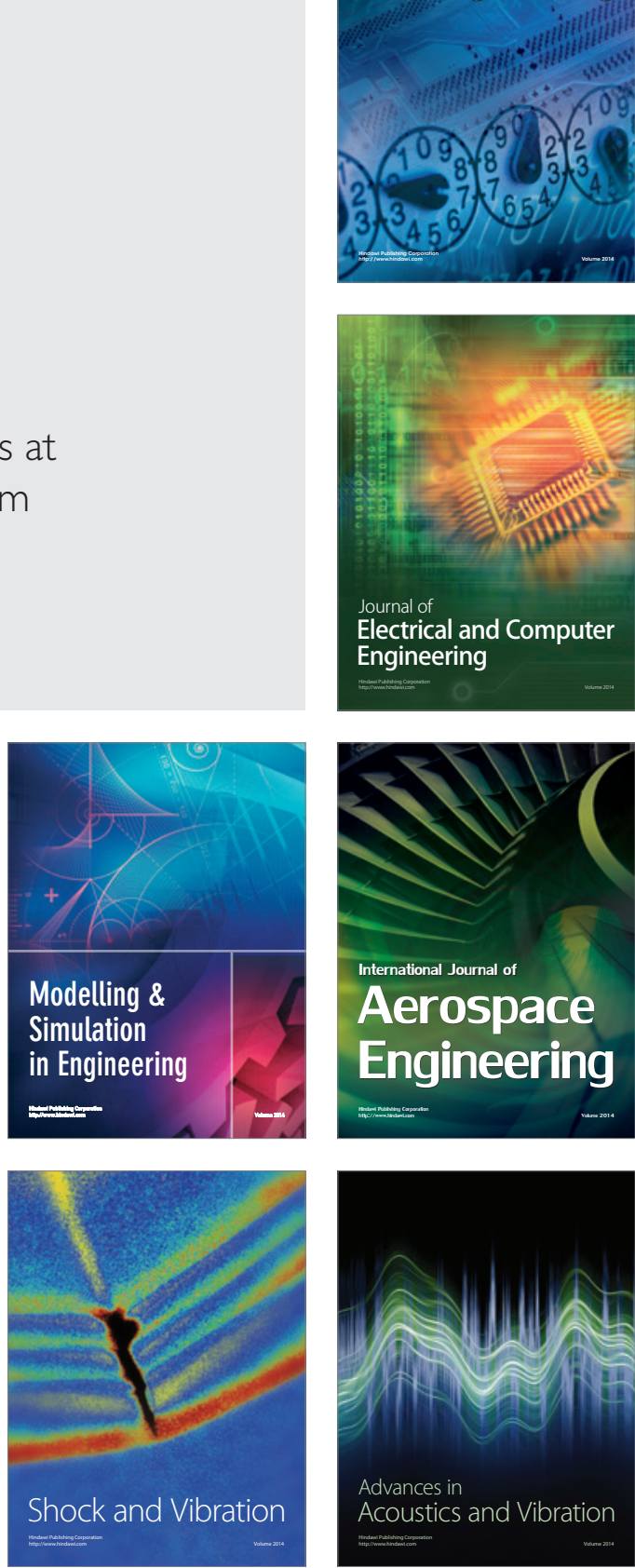